\title{
Aero-Optical and Flow Measurements Over a Flat-Windowed Turret
}

\author{
Stanislav Gordeyev* \\ University of Notre Dame, Notre Dame, Indiana 46556 \\ Tim E. Hayden \pm \\ U.S. Air Force Academy, Colorado Springs, Colorado 80840 \\ and \\ Eric J. Jumper \\ University of Notre Dame, Notre Dame, Indiana 46556 \\ DOI: $\underline{10.2514 / 1.24468}$
}

\begin{abstract}
An extensive investigation of the aberrating character of flow over a hemisphere-on-cylinder turret with a flat window was performed. Optical distortions over the window were measured using a two-dimensional wave front sensor and a Malley probe. The Malley probe measurements were complemented with simultaneous hot-wire measurements of the velocity field normal to the window at several points across its diameter. The tests were run for a fixed elevation for several azimuthal angles over a range of Mach numbers. The results provide the levels of unsteady optical aberration across the window, as well as the local thickness, intensity, and convective speed of the separated flow over the window. Results reveal that the optical distortions grow approximately with the square of the incoming Mach number multiplied by the freestream density, $\mathrm{OPD}_{\mathrm{rms}} \sim \rho M^{2}$, as well as with the changing character of the aberrating flow with azimuth angle.
\end{abstract}

\section{Nomenclature}

$\begin{array}{ll}\text { Ap } & =\text { laser beam round aperture diameter } \\ \text { Corr } & =\text { velocity-jitter signal correlation function } \\ E\{\} & =\text { time/ensemble averaging procedure } \\ F & =\text { focal length } \\ f & =\text { frequency } \\ I & =\text { far-field point-spread function } \\ M & =\text { Mach number } \\ \mathrm{OPD} & =\text { optical path difference } \\ \mathrm{OPD} & =\text { root mean square of optical path difference } \\ R & =\text { flat-mirror radius } \\ S & =\text { jitter signal cross-correlation function } \\ s & =\text { beams spacing } \\ T & =\text { block sampling time } \\ t & =\text { time } \\ U_{c} & =\text { convective speed } \\ U_{\mathrm{free}} & =\text { freestream speed } \\ W & =\text { wave front } \\ u & =\text { streamwise velocity component } \\ x, y, z & =\text { turret coordinate system } \\ \alpha, \alpha_{1}, \alpha_{2} & =\text { far-field coordinates } \\ \Delta & =\text { beam displacement } \\ \delta_{1}, \delta_{2} & =\text { jitter signals } \\ \theta & =\text { azimuthal angle } \\ \lambda & =\text { laser wavelength } \\ \rho & =\text { freestream density } \\ \rho_{\mathrm{SL}} & =\text { sea-level density, } \rho_{\mathrm{SL}}=1.229 \mathrm{~kg} \cdot \mathrm{m}^{-3} \\ & \end{array}$

Presented as Paper 3074 at the 37th AIAA Plasmadynamics and Lasers Conference, San Francisco, California, 5-8 June 2002; received 7 April 2006; revision received 14 August 2006; accepted for publication 27 September 2006. Copyright (C) 2006 by S. Gordeyev, T. Hayden, and E. Jumper. Published by the American Institute of Aeronautics and Astronautics, Inc., with permission. Copies of this paper may be made for personal or internal use, on condition that the copier pay the $\$ 10.00$ per-copy fee to the Copyright Clearance Center, Inc., 222 Rosewood Drive, Danvers, MA 01923; include the code $\$ 10.00$ in correspondence with the CCC.

*Assistant Research Professor, Department of Aerospace and Mechanical Engineering. Member AIAA.

${ }^{\dagger}$ Aerospace Engineer, Aeronautics Department. Senior Member AIAA.

†Professor, Department of Aerospace and Mechanical Engineering. Fellow AIAA.

\section{Motivation}

W HEN an otherwise-collimated laser beam passes through a variable-index-of-refraction turbulent flow, its wave front becomes dynamically aberrated (unsteady). These aberrations degrade the beam's ability to be focused in the far field, thereby reducing the system utility of the beam that may be used for communication, interrogation, and targeting or as a directed-energy weapon. When the laser platform is an aircraft, the two main causes of beam degradation are the thin layer and immediate airflow around the aircraft, referred to as the aero-optic problem [1], and the intervening, orders-of-magnitude-longer propagation path through the atmosphere to the target, referred to as the atmosphericpropagation problem. Modern beam-control, adaptive-optic methods appear to now be able to mitigate the atmosphericpropagation effects on the beam; however, both the spatial and temporal bandwidths of the aero-optic problem place it well outside the capabilities of these traditional approaches [2]. It has only been a decade since the first time-resolved wave front measurements for propagation through a relevant aero-optic flowfield were made [3]; before that time, aero-optic propagation environments were characterized by limited time-unresolved interferograms and indirectly inferred from hot-wire anemometry techniques [1,2]. In general, the paucity of such characterizations that were available treated the aero-optic problem as a stochastic problem and reduced the measurements to very unspecific measures of optical degradation such as $\mathrm{OPD}_{\mathrm{rms}}$. Such measures, although providing an estimation of the degradation that might be expected, provided little in the way of higher-order information about the aberrating environment's aberration coherence length (spatial bandwidth) and temporal bandwidth over relevant laser-beam apertures. The lack of such characterizations made it impossible to either infer the far-field degradation in the point-spread function or address the requirements for adaptive-optic mitigation schemes.

The ability to collect copious spatial and temporal wave front information through relevant aero-optical-type flowfields changed abruptly with the invention by Malley et al. [4] of a new approach to interrogating these fields with a direct optical method that used a single, small-aperture laser beam at a single location in the larger aperture. Malley at al. exploited the fact that aberrations due to convecting optically active turbulence themselves convect through the aperture. This approach was further enhanced with the 
introduction of multiple beams over the aperture to construct an actual time-resolved one-dimensional wave front sensor in the flow direction: the small-aperture-beam technique (SABT) [5]. As more experience was gained by investigating aero-optical flowfields, it became apparent that returning to configurations more akin to the Malley et al. original idea (of a single interrogation location over an aperture) provided an extremely powerful aero-optical measurement tool. We introduced a new, two-small-aperture-beam instrument (and accompanying analysis) wave-front-characterization instrument that we refer to as a Malley probe [6]. This instrument represented a new milestone in the ability to extract aero-optical and flow information from a single, simple-to-use instrument [7]. In a companion paper [8], the Malley probe described in [6] is redescribed, and a comparison with wave front data from other instruments is given.

Although the methods used to characterize aero-optical flowfields before a decade ago lacked higher-order information, such little information that was available provided many instances of propagation through flows around geometries that might be used as relevant aircraft-protruding beam directors (see [9], for example). On the other hand, although more recent, time-resolved wave-front studies available in the open literature are at relevant Mach and Reynolds numbers, they are for fundamental flows like planar free shear layers $[2,3]$ or canonical attached turbulent boundary layers [6]. This paper changes that trend and presents aero-optical data for a generic beam director, a hemisphere-on-cylinder turret with a flat window, similar to earlier geometries found in [9], for example. Such turrets, unlike a hemisphere mounted flush with the skin of the platform, provide a better field of regard and are currently being used on the Predator UAV, for example. Typical beam apertures for these turrets are approximately one-third of the turret diameter. The data presented here were collected for three viewing angles; all these angles are at an elevation of $30 \mathrm{deg}$, one at a 90-deg angle back from the incoming flow, one at $100 \mathrm{deg}$, and the last for $110 \mathrm{deg}$. Because the beam director has a flat "window" over the aperture, these angles represent a weak separation, with reattachment near the leading edge of the aperture; a weakly attached, "nearly separated" flow over the aperture; and separated flow over the aperture, respectively. Unlike the fundamental-flow studies referenced earlier, in which optical data were amenable to straightforward interpretation, the data in this paper are complicated and, like the data of previous decades, difficult to interpret. Albeit difficult to understand, the data represent a fairly comprehensive collection of complementary types of data. As mentioned previously, the bulk of the optical data were collected using a Malley probe; however, a complementary set of non-timeresolved wave fronts over the full aperture was collected. Also, for the first time, simultaneous hot-wire measurements were made with the Malley probe data. Although the overall interpretation of the optical/flow environment still presents an incomplete picture of exactly what is going on, these new Malley probe/hot-wire data reveal some interesting physics about the role that a recirculation region over the aperture plays in the overall optical environment.

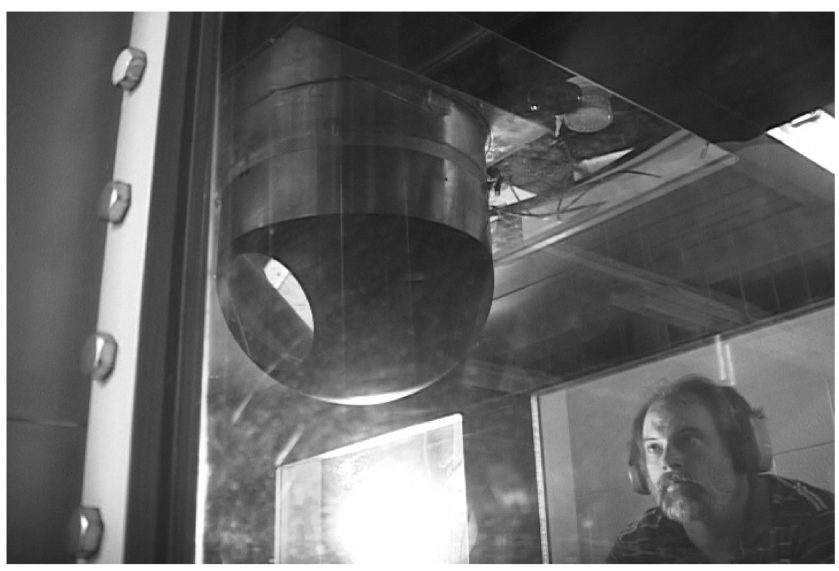

Fig. 1 The turret assembly installed in the wind tunnel.

\section{Experimental Setup}

All measurements were performed in a closed-loop, subsonic wind tunnel located at the U.S. Air Force Academy in Colorado Springs, Colorado. The tunnel cross section is $3 \times 3 \mathrm{ft}$, and the Mach number range is from 0 to 0.6 . The tunnel has three $1 \times 1 \mathrm{ft}$ optical access windows on the front and back tunnel walls and is equipped with a three-dimensional traverse system. The tunnel elevation is $7160 \mathrm{ft}$ above sea level, and the total density of the tunnel flow was measured to be $0.96 \mathrm{~kg} / \mathrm{m}^{3}$ during the measurements.

Wind-tunnel tests were conducted on the hemisphere-on-cylinder turret model, shown mounted in the roof of the Academy tunnel in Fig. 1. The flat window of the 12-in.-diam hemispherical portion of the turret could be rotated from 25 to $90 \mathrm{deg}$ of elevation and the base could be rotated through 360 deg of azimuth (see Fig. 2); although, for these tests, only three azimuthal and elevation combinations were used. Unique to these tests, the turret window was created by inserting a 5.4-in. first-surface mirror into the hemispherical portion of the turret so that the mirror's edge was flush with the hemispherical surface. The mirror surface was flat to less than one-tenth of a wave at $630 \mathrm{~nm}$ (cf. Sec. III.B). The slope discontinuity between the sphere and the mirror is $27.5 \mathrm{deg}$. The 12 -in.-diam cylindrical base was 4 -in. tall. The turret was sealed to the laboratory, so that the interior of the beam director was nominally at the test-section static pressure.

The beam director's system of coordinates is shown on the left in Fig. 2. The origin is placed at the center of the flat mirror. The $x$ and $y$ axes lie in the mirror's front-surface plane, with the $x$ axis aligned horizontally and the $\mathrm{y}$ axis being normal to the $\mathrm{x}$ axis. The $\mathrm{z}$ axis is normal to the mirror surface.

As mentioned in the Introduction, the flow over the turret was studied for one elevation angle of $30 \mathrm{deg}$ and three azimuthal angles $(\theta=90,100$, and $110 \mathrm{deg})$ relative to the flow heading. Thus, the mirror was either faced normal to the incoming flow (90 deg) or slightly downstream (100 and $110 \mathrm{deg}$ ). Various Mach numbers were

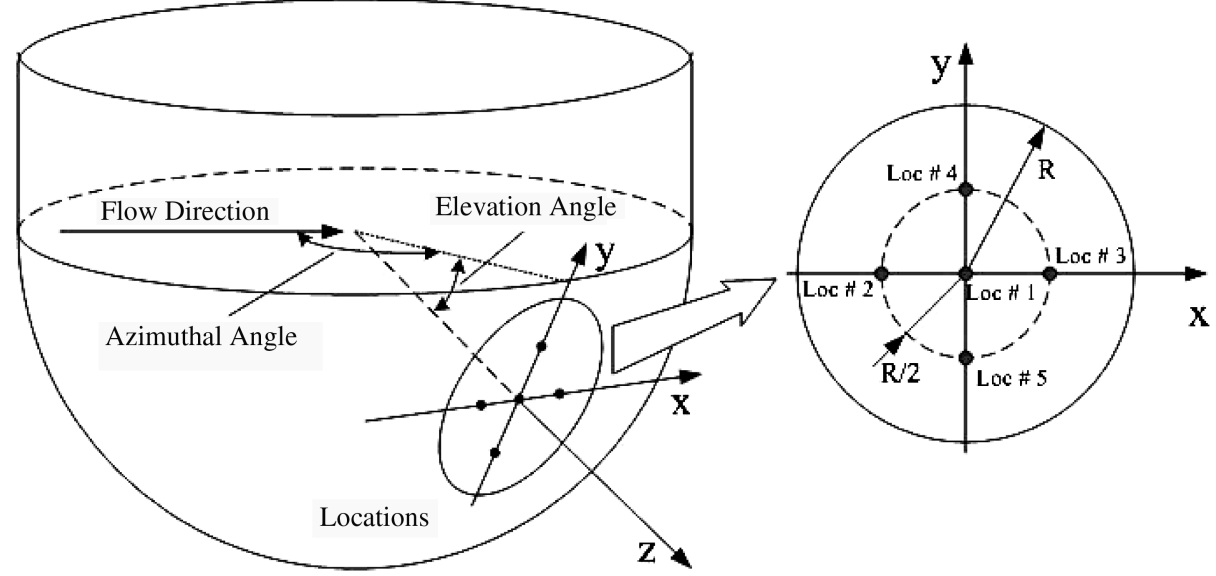

Fig. 2 Turret's system of coordinates (left) and velocity and Malley probe measurement locations at the mirror (right). 

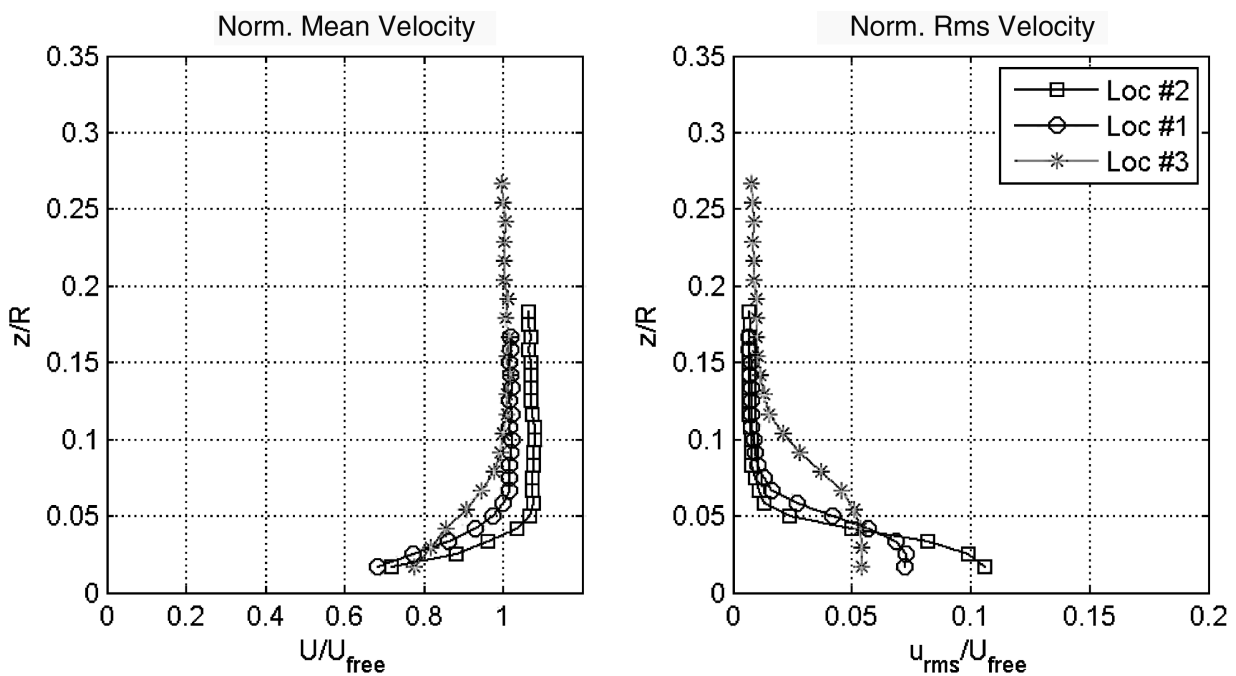

Fig. 3 The mean and fluctuating velocity profiles at three locations at the mirror for $M=0.35$ for the azimuthal angle of 100 deg. Velocities are normalized by the freestream speed.

studied ranging from $M=0.3$ to 0.5 . The spatial distribution of optical aberrations in the plane of the mirror's surface was measured using a commercially available 2-D wave front sensor, and extensive studies of optical aberrations at five locations on the mirror were measured using a Malley probe (shown on the right in Fig. 2). To characterize the flowfield, a single hot wire was used to obtain velocity profiles normal to the mirror at three of the five locations shown in Fig. 2 for two azimuthal angles of 100 and 110 deg. In addition, to determine the location of the optically active structures along the optical path normal to the mirror, simultaneous velocity and optical aberrations were measured with the hot wire at a range of $\mathrm{z}$ locations, for two locations over the mirror, at a Mach number of 0.37 , for one azimuthal angle of $110 \mathrm{deg}$, using a single hot wire and the Malley probe.

\section{Results}

\section{A. Hot-Wire Probe}

The velocity profiles in the normal direction were taken for $M=0.35$ for two azimuthal angles of 100 and $110 \mathrm{deg}$ for locations 2, 1, and 3, indicated on the right in Fig. 2. Data were collected at a sampling rate of $100 \mathrm{kHz}$ for $10 \mathrm{~s}$. Results for $100 \mathrm{deg}$ are shown in Fig. 3. For 100 deg of azimuth, the flow separates at the leading edge of the mirror. Because the separated flow faced only a mild adverse pressure gradient from the 10-deg, back-facing mirror surface, the separation caused the flow to trip, facilitating a reattachment as a turbulent boundary layer and forming a weak separation bubble at the mirror's leading edge. Thus, the velocity profiles in Fig. $\underline{3}$ show the presence of a boundary-layer-like flow over the mirror, with growing thickness and relatively low normalized turbulence level (from 0.05 to 0.1 ) that decays with increasing downstream distance.

Results for $110 \mathrm{deg}$ are presented in Fig. 4. At $110 \mathrm{deg}$, the flow now faces a stronger adverse pressure gradient over a 20-deg backfacing mirror and clearly remains separated over the mirror. The velocity profiles exhibit a shear-layer-like behavior, with a high speed being the freestream speed and the low speed being around 0.4 of the freestream speed. The shear layer thickness grows downstream, indicating the formation of shear-layer, KelvinHelmholtz-driven, coherent structures. The turbulence levels have a single peak in the normal direction, with maximum turbulence levels of 0.2 of the freestream speed, which is two-four times higher than for the 100-deg case.

\section{B. Two-Dimensional Wave Fronts}

Our approach of using a high-quality, optically flat, first-surface mirror as a surrogate beam-director window was to avoid the complications of injecting and/or extracting laser beams through the beam director. This allowed for a more utilitarian approach to collecting both the 2-D wave fronts and the Malley probe data. The
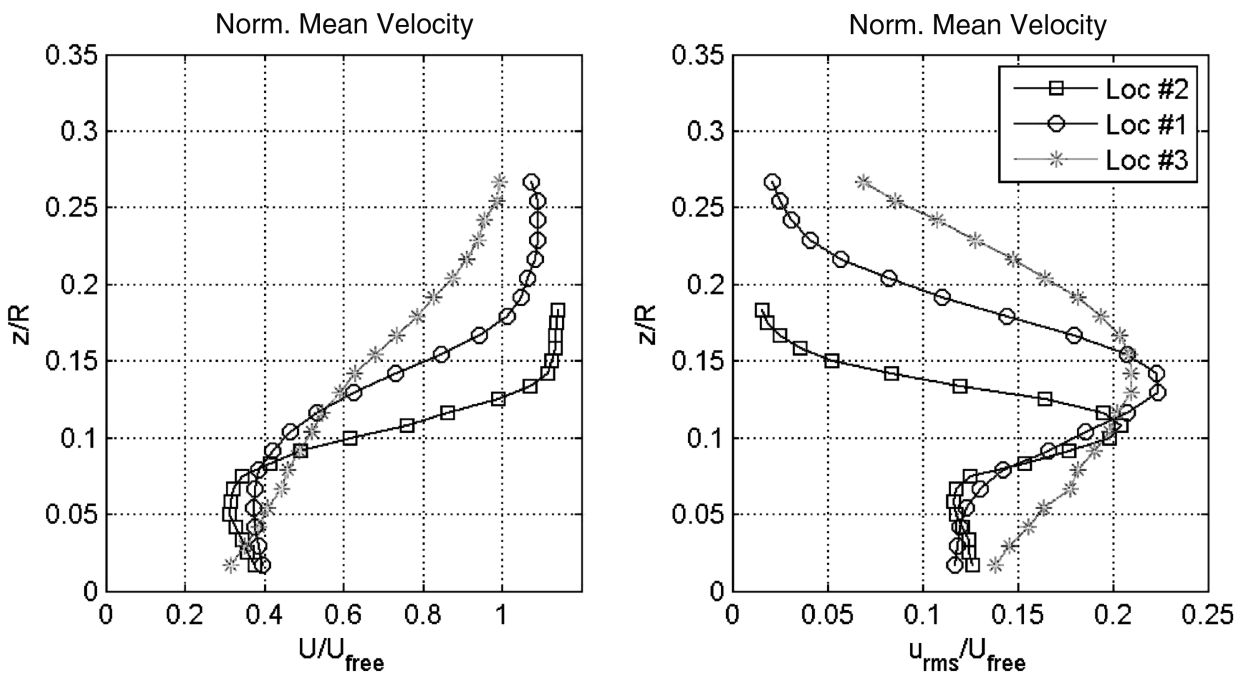

Fig. 4 The mean and fluctuating velocity profiles at three locations at the mirror for $M=0.35$ for the azimuthal angle of 110 deg. Velocities are normalized by the freestream speed. 


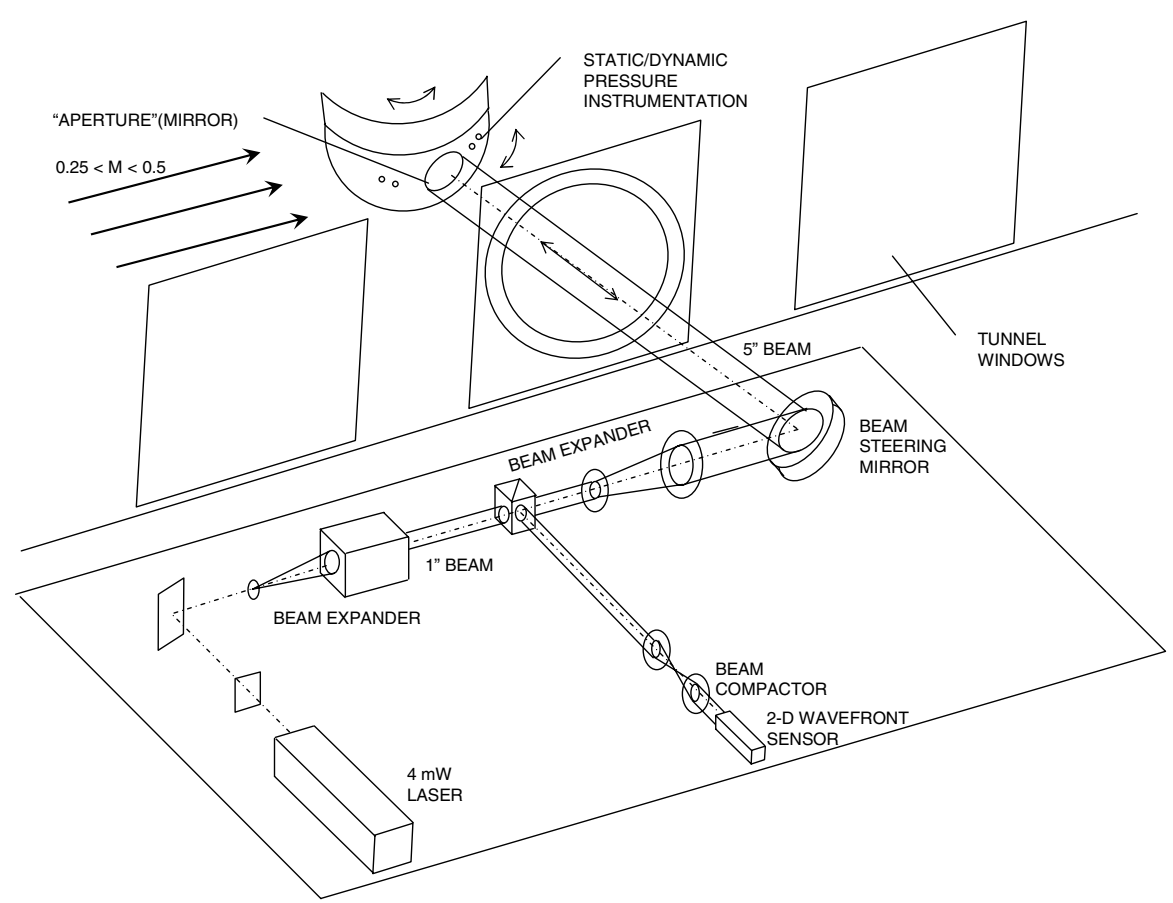

Fig. 5 Two-dimensional wave front experimental setup.

approach allowed all the optical components, including the beamsteering mirrors and various laser sources and sensors, to share a single optical bench. The optical setup is shown schematically in Fig. 5.

For the wave front measurements, a 4-mW CW He-Ne laser was expanded to a 1-in. collimated beam, which in turn was expanded to a 4-in. beam. The 4-in. beam was steered into the wind tunnel test section normal to the mirror. The turret mirror reflected the beam coaxially back onto the optical bench, where it was split using a beam-splitter cube. Thus, the beam went through the turbulent flow twice, doubling the signal-to-noise ratio of the aberration measurement. Finally, after a pair of contracting lenses, the beam's optical distortions were measured using a 2-D Hartmann-Shack wave front sensor with a $33 \times 44$ lenselet array, mounted in front of a CCD camera. The exposure time was $1 / 10,000$ of a second. The wave fronts were sampled at a $30-\mathrm{Hz}$ sampling rate. Several hundred wave fronts were recorded for each case.

The wave fronts were postprocessed as follows:

1) Each wave front $W(x, y, t)$ was constructed as a change from the reference no-flow wave front to eliminate optical aberrations imposed on the collimated laser beam by the optical setup.

2) In general, a wave front $W(x, y, t)$ can be expanded in Taylor series as

$$
W(x, y, t)=A+B_{1}(t) x+B_{2}(t) y+W_{\text {HighOrder }}(x, y, t)
$$

where $A$ is referred to as a piston component and $B_{1}(t)$ and $B_{2}(t)$ are instantaneous tip/tilt components.

3) The piston component and instantaneous tip/tilt were removed from each wave front.

4) A steady wave front (steady-lensing effect) was computed by averaging all resulting wave fronts in time

$$
W_{\text {steady }}(x, y)=E\left\{W_{\text {HighOrder }}(x, y, t)\right\}
$$

and it was also removed from each wave front.

5) The final wave fronts [for simplicity, denoted again as $W(x, y, t)]$ contain only the unsteady tilt-removed aberrations. They reveal the character of the convective, optically active structures passing over the aperture.

Although instantaneous tip/tilt information is important for adaptive and corrective optics systems, as well as for understanding flow physics, because these could not be easily separated from combined tunnel/model/optical-table vibrations, which also manifest themselves as tip/tilt, we were forced to remove tip/tilt altogether from the data and study only the unsteady portion of wave fronts.

Two representative, instantaneous wave fronts $W(x, y, t)$, as treated previously, for a 110-deg look-back angle for $M=0.3$ and 0.5 are shown in the upper two frames of Fig. 6. Also shown in the lower frames of Fig. $\underline{6}$ are these two wave fronts' far-field pointspread functions $I\left(\alpha_{1}, \alpha_{2}\right)$ for $\lambda=1 \mu \mathrm{m}$. The far-field point-spread functions were calculated assuming top-hat beam intensity (constant beam intensity inside the aperture and zero beam intensity outside the aperture) using the equation

$$
I\left(\alpha_{1}, \alpha_{2}\right)=\left|\frac{\iint_{\mathrm{Ap}} \exp \left(2 \pi i\left[\frac{W(x, y)-\left(\alpha_{1} x+\alpha_{2} y\right)}{\lambda}\right]\right) \mathrm{d} x \mathrm{~d} y}{\iint_{\mathrm{Ap}} \mathrm{d} x \mathrm{~d} y}\right|^{2}
$$

Although the presented wave fronts are single realizations, they are representative of all the wave fronts recorded at these conditions. Their topologies are consistent with the inferences drawn from the hot-wire data and they suggest that optically active, crossflowelongated, shear-layer-like structures form and convect over the aperture.

To characterize the level of optical distortions, the spatial rms optical path difference over the aperture was calculated for each wave front, and a time-averaged $\mathrm{OPD}_{\mathrm{rms}}$ was found for each test case:

$$
\mathrm{OPD}_{\mathrm{rms}}=E\left\{\sqrt{[\mathrm{OPD}(x, y, t)-\overline{\mathrm{OPD}(x, y, t)}]^{2}}\right\}
$$

where $\operatorname{OPD}(x, y, t)=-W(x, y, t)$ and the bar denotes a spatial averaging. Far-field patterns normalized by the diffraction-limited intensity were also computed for a wavelength of $\lambda=1 \mu \mathrm{m}$, using Eq. (1).

Levels of optical aberrations increase with the Mach number, resulting in a breaking up of the intensity in the flow direction and reducing the peak intensity in the far-field pattern. A summary of $\mathrm{OPD}_{\mathrm{rms}}$ for all measured cases is presented in Table 1 . Clearly, the optical aberrations increase with the increasing azimuthal angle, and the aberrations increase with increasing Mach numbers at every angle. The far-field intensity values reported in the far-field frames in Fig. 6 are normalized by the diffraction-limited, on axis, with ideal 

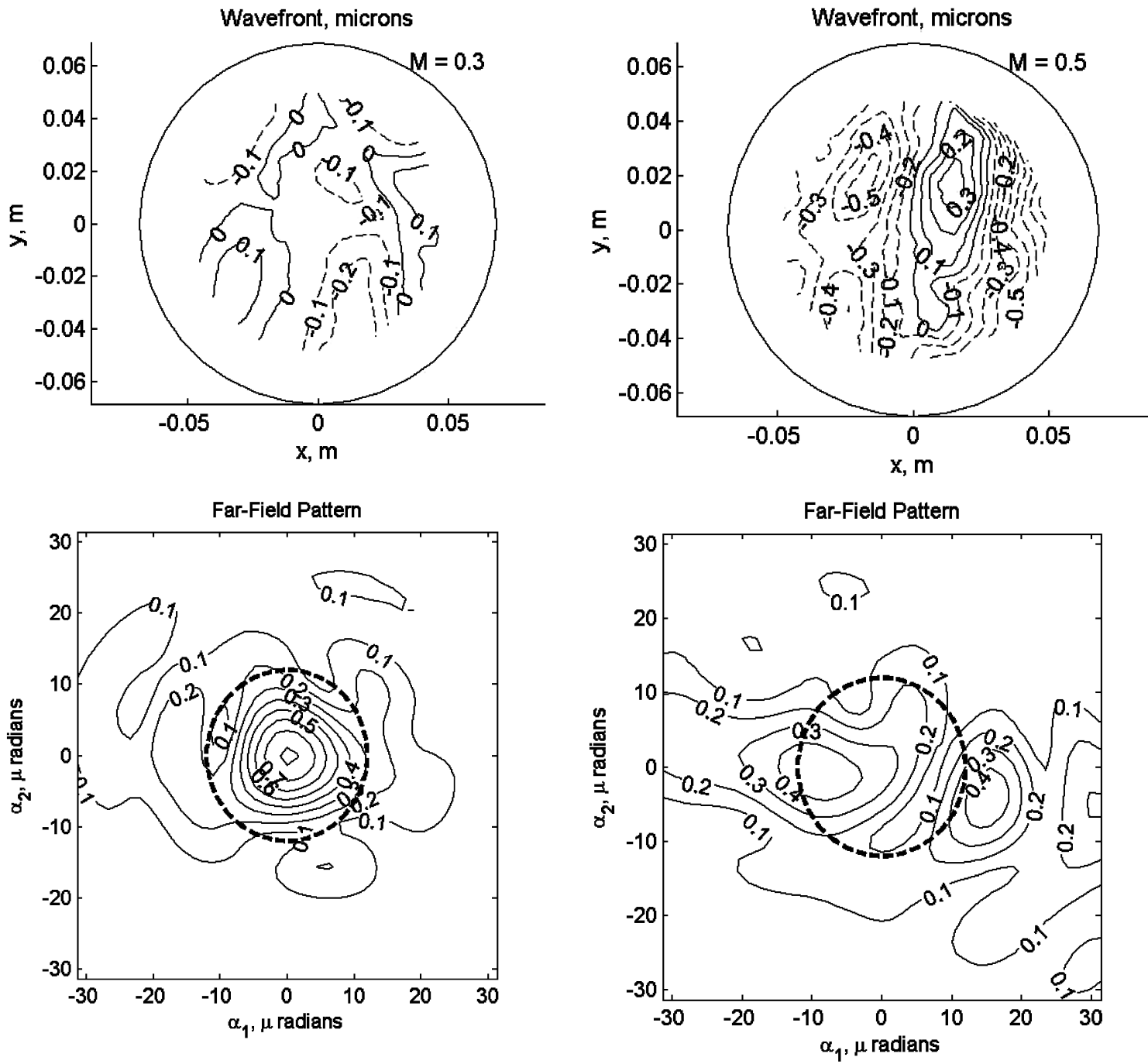

Fig. 6 Wave fronts (upper plots) and far-field patterns at $1-\mu \mathrm{m}$ wavelength (lower plots) for $M=0.3$ and $M=0.5$. The azimuthal angle is 110 deg. The flow goes from left to right. The mirror is represented by a black circle. The far-field Airy disc is marked with a thick dashed line.

intensity given by

$$
I_{\text {ideal }}\left(\alpha=\sqrt{\alpha_{1}^{2}+\alpha_{2}^{2}}\right)=\left[J_{1}\left(\frac{\alpha \mathrm{Ap}}{2 \lambda}\right) /\left(\frac{\alpha \mathrm{Ap}}{\lambda}\right)\right]^{2}
$$

with $\alpha=0.0$. The location of the so-called Airy disc $\left(\alpha_{\text {Airy }}=\right.$ $1.22 \lambda / \mathrm{Ap})$, is marked on far-field patterns in Fig. 6 as a thick dashed line. At $M=0.5$, optical aberrations become strong enough to fractionate the beam's focus.

\section{Malley Probe}

To obtain time-resolved unsteady wave front data, the Malley probe was used to measure optical aberrations at five locations over the aperture (mirror), shown on the right in Fig. 2. The Malley probe is an optical instrument that can make direct, accurate measurements of dynamically distorting wave fronts, including the characteristics of the $\operatorname{OPD}(t)$. By moving the instrument, an entire large aperture can

Table 1 OPD $_{\text {rms }}$ results from 2-D wave front sensor

\begin{tabular}{lcc}
\hline \hline$\theta$, deg & $M$ & OPD $_{\text {rms }}, \mu \mathrm{m}$ \\
\hline 90 & 0.3 & 0.0254 \\
& 0.4 & 0.0383 \\
100 & 0.3 & 0.0577 \\
& 0.4 & 0.0847 \\
110 & 0.3 & 0.0653 \\
& 0.4 & 0.0856 \\
& 0.5 & 0.0985 \\
\hline \hline
\end{tabular}

be optically characterized. This characterization includes not only the measurement of $\mathrm{OPD}_{\mathrm{rms}}$, but also the spatial and temporal frequencies of the aberrations. The instrument itself is a further development by Notre Dame of an instrument described in a paper by Malley, et. al. [4] and has now been shown to give extremely accurate measurements of $\mathrm{OPD}_{\text {rms }}[6,8,10-14]$. The instrument consists of two closely spaced, small-aperture (typically, 1_mm) beams ( $\sim 3-10 \mathrm{~mm}$ apart) that are aligned, front beam to aft beam, in the streamwise direction (see Fig. 7); the second beam is used to extract convection velocity information contained on the beam-deflection angles by cross-correlating them and obtaining the time delay between the signals. Knowing the displacement between the beams and this delay time, the convective velocity can be computed. As described in [5] the deflection angle of the small aperture beam is the negative spatial gradient of the OPDat the probe-beam location, $\delta(x, y, t)=\operatorname{grad} W(x, y, t)=-\operatorname{grad} \operatorname{OPD}(x, y, t)$. The instantaneous deflection-angle measurement procedure is presented schematically in Fig. 8. A small-aperture laser beam passes through a turbulent flow of interest and gets deflected. The laser beam is then focused on a position sensing device, labeled as PSDs in Fig. 7 (we used lateral effect detectors), which accurately measures the beam's centroid position $\Delta(t)$ as a function of time. The deflection angle is calculated as $\delta(t)=\Delta(t) / F$. Because the position-sensing device is an analog photosensitive crystal with a response time of less than $1 \mu \mathrm{s}$, it allows sampling deflection angles at high sampling rates, on the order of hundreds of kilohertz.

To compute the convective speed, deflection signals are crosscorrelated to find a time delay between the signals. One way to do this 


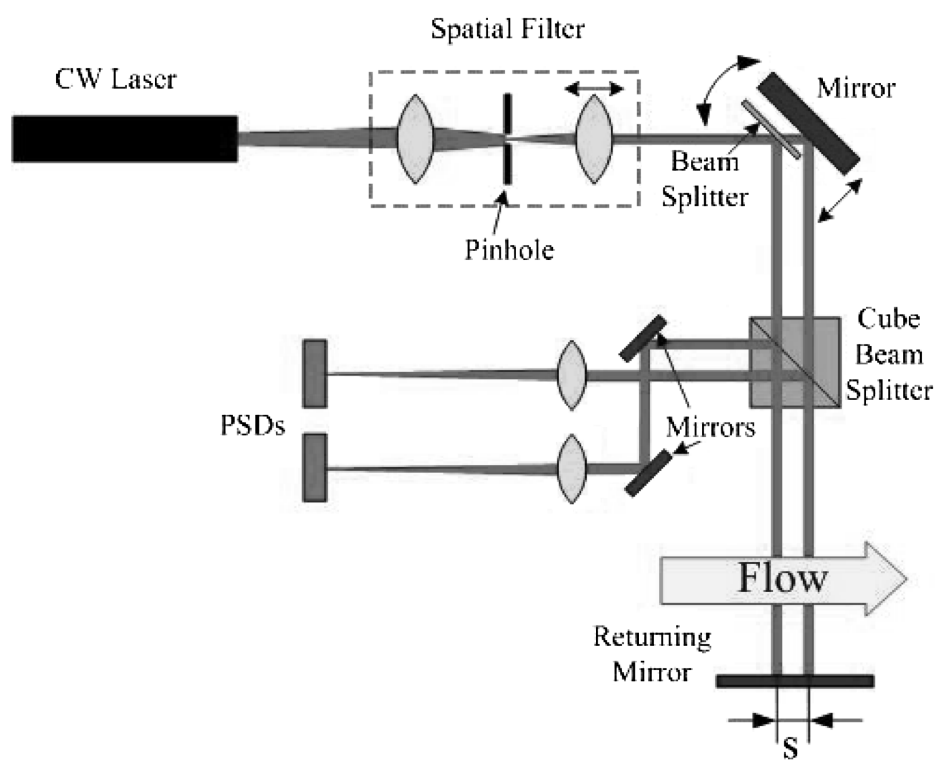

Fig. 7 Malley probe schematic.

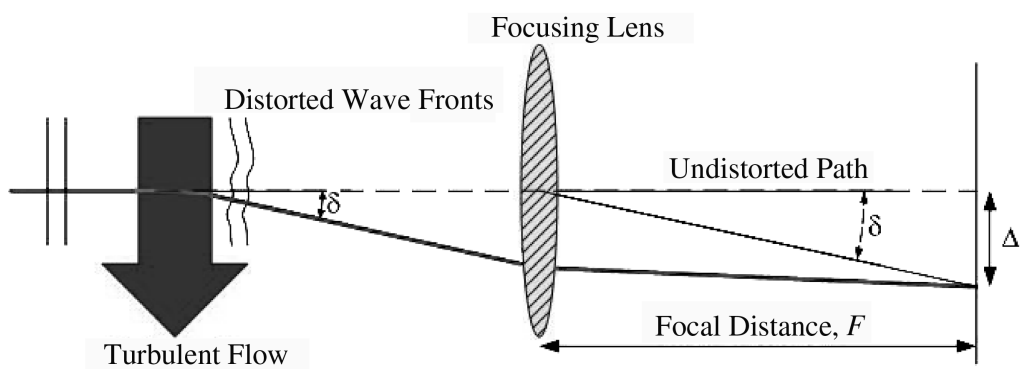

Fig. 8 Principle of deflection-angle measurements.

is to compute a time-delayed correlation function $R(\tau)=E\left\{\delta_{1}(t) \times\right.$ $\delta_{2}(t+\tau)$ \} between the two signals $\delta_{1}(t)$ and $\delta_{2}(t)$ from the two probe beams (direct method). $U_{c}$ is computed knowing a separation $s$ between beams and a time delay of the highest correlation, $\tau^{\max }$, $R\left(\tau^{\max }\right)=\max$, that is, $U_{c}=s / \tau^{\max }$. The problem with the direct approach is that it is sensitive to signal contamination (such as mechanical vibration of the return mirror and electronic noise, for instance). Another way of computing the time delay is to use a spectral method, proposed in [6]. In this approach, that is, to analyze a spectral cross-correlation function,

$$
S(\omega)=\int R(\tau) \exp (-i \omega \tau) \mathrm{d} \tau=\frac{1}{T}\left\langle\hat{\delta}_{1}(\omega) \hat{\delta}_{2}^{*}(\omega)\right\rangle
$$

is used, where the brackets denote an ensemble average. In the case of a pure convecting structure, the signal $\delta_{2}$ downstream is just a timedelayed signal of the upstream signal $\delta_{2}(t)=\delta_{1}\left(t-\tau^{\max }\right)$. The Fourier transform of $\delta_{2}$ becomes $\hat{\delta}_{2}(\omega)=\hat{\delta}_{1}(\omega) \exp \left(-i \omega \tau^{\max }\right)$. Using this relation, the expression for the spectral correlation $S(\omega)$ becomes

$$
\begin{aligned}
& S(\omega)=1 / T\left\langle\hat{\delta}_{1}(\omega) \hat{\delta}_{2}^{*}(\omega)\right\rangle=1 / T\left\langle\hat{\delta}_{1}(\omega)\left[\hat{\delta}_{1}(\omega) \exp \left(-i \omega \tau^{\max }\right)\right]^{*}\right\rangle \\
& \quad=A(\omega) \exp \left(i \omega \tau^{\max }\right)
\end{aligned}
$$

where $A(\omega)=1 / T\left\langle\hat{\delta}_{1}(\omega) \hat{\delta}_{1}^{*}(\omega)\right\rangle$ is a real function of $\omega$. Thus, by analyzing the slope of the argument of the spectral cross-correlation function, one can find the time delay $\tau^{\max }$ :

$$
\tau^{\max }(f)=\frac{1}{2 \pi} \frac{d}{\mathrm{~d} f} \arg [S(f)]
$$

The spectral method allows one to extend convective velocity measurements for the case, then the convective speed is a function of frequency, $U_{c}(f)=\mathrm{s} / \tau^{\max }(f)$. This information is very useful, for instance, when the beams encounter the flow with different optically active regions, and it is still possible to measure convective speeds for each region using the spectral method, as long as optical structures associated with each region have distinct frequency bands [10].

Optical $\operatorname{OPD}\left(t, x_{0}\right)$ can be reconstructed from the jitter signals in the $\mathrm{x}$ direction using the Taylor frozen field hypothesis:

$$
\begin{gathered}
\operatorname{OPL}\left(t, x_{0}\right)=-U_{c} \int^{t} \delta_{1}(t) \mathrm{d} t \\
\operatorname{OPD}\left(t, x_{0}\right)=\operatorname{OPL}\left(t, x_{0}\right)-\overline{\operatorname{OPL}\left(t, x_{0}\right)}
\end{gathered}
$$

Based on preliminary measurements of the temporal frequencies present in the dynamic aberrations, data were sampled at $100 \mathrm{kHz}$ for $10 \mathrm{~s}$. A beam separation of $5 \mathrm{~mm}$ in the streamwise direction was used.

Phase plots of $\arg [S(f)]$ vs frequency at the location at the center of the mirror (location 1) for two azimuthal angles of 100 and $110 \mathrm{deg}$ for $M=0.4$ are presented in Fig. 9. The phase values hover around zero up to $\sim 1 \mathrm{kHz}$ and then become linearly increasing with frequencies above $1 \mathrm{kHz}$. This indicates that jitter data below $1 \mathrm{kHz}$ are due to stationary effects such as vibrations, stationary-lensing, separation-bubble breathing, or far-field effects of the necklace vortex around the base of the turret, discovered for similar turret geometries [11]. No doubt, stationary optical aberrations carry important information about the aberrating character of the flow; however, for the stationary effects, the phase $\arg [\mathrm{S}(f)]$ is zero, and the computed convective speeds, as stated previously, are formally infinite. Thus, stationary effects cannot be unfolded and studied using the convective assumption embedded in Eq. (6). For this study, all stationary effects were filtered from the data. Although a Malley probe offers an accurate measure of the convective component of the 
$\theta=100$ degrees

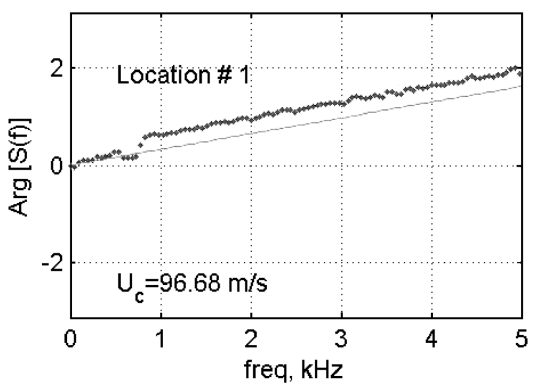

$\theta=110$ degrees

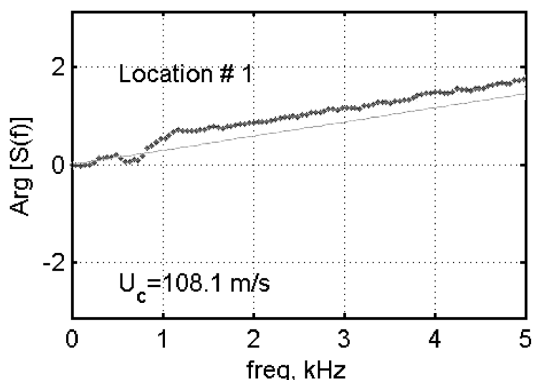

Fig. 9 Phase plots $\arg S(f)$ for location 1 for the azimuthal angles of 100 (left) and 110 (right) deg at $M=0.4$.

aberrating signal, other instruments should be used to investigate the nature of the stationary structures $[\underline{8}, 11]$. Above $1 \mathrm{kHz}$, the phase slope is nonzero and its slope can be used to compute the mean convective speed of optically active structures.

By high-pass filtering the Malley probe data at $1 \mathrm{kHz}$, any stationary aberrations were removed from the signals before computing the convective speeds and OPDs for each case. Results of greater than $1-\mathrm{kHz}$ convective speed and reconstructed OPDs for locations 1, 3, 4, and 5 are given in Fig. 10. Convective speeds, presented in Fig. 10, were in a range from 0.6 to 0.8 of the freestream speed $U_{\text {free. }}$ These values of convective speed, obtained nonintrusively using the Malley probe, are consistent with hotwire data, presented in Figs. 3 and 4 . For boundary layers, the convective speed should be $0 . \overline{8}$ of the freestream velocity [6]; for shear layers, the convective speed is the half-sum of the speeds above and below the shear layer, thus, in this case, giving $\sim 0.75$ of the freestream speed.

Having the jitter data at each location, time and position can be exchanged using a frozen field hypothesis, $x=-U_{c} t$, to project the
Malley probe data into a pseudo wave front in the up- and downstream direction [8], $\operatorname{OPD}(x)=\operatorname{OPD}\left(t=-x / U_{c}\right)$. This allows for the construction of a time series of wave fronts over any aperture size. As described in detail in [12], when tip/tilt and piston components are removed over the aperture, the resulting OPD changes as a function of aberration frequency and aperture size. To take this into account, the Malley-probe-unapertured (that is, for infinite aperture) OPDs from Eq. (6) were 4-in.-apertured to better compare to the 4-in. aperture, $2-\overline{\mathrm{D}}$ wave front data described in Sec. III.B. After extending data to a 4-in. aperture, every realization had local piston and tilt removed, frame by frame, similar to the 2-D wave front tilt-removing procedure outlined previously. The subsequent wave front histories then had $\mathrm{OPD}_{\text {rms }}$ computed over the aperture, frame by frame, and $\mathrm{OPD}_{\mathrm{rms}}$ was then ensemble-averaged over the entire record. The resulting average aberrations are presented in Fig. 10. Freestream densities for $M=0.3,0.4$, and 0.5 were $\rho=0.92,0 . \overline{88}$, and $0.85 \mathrm{~kg} / \mathrm{m}^{3}$, respectively. $\mathrm{OPD}_{\mathrm{rms}}$ is lower for the 90-deg azimuthal angle case and higher for the 100 and 110deg cases, which is consistent with the 2-D wave front measurements
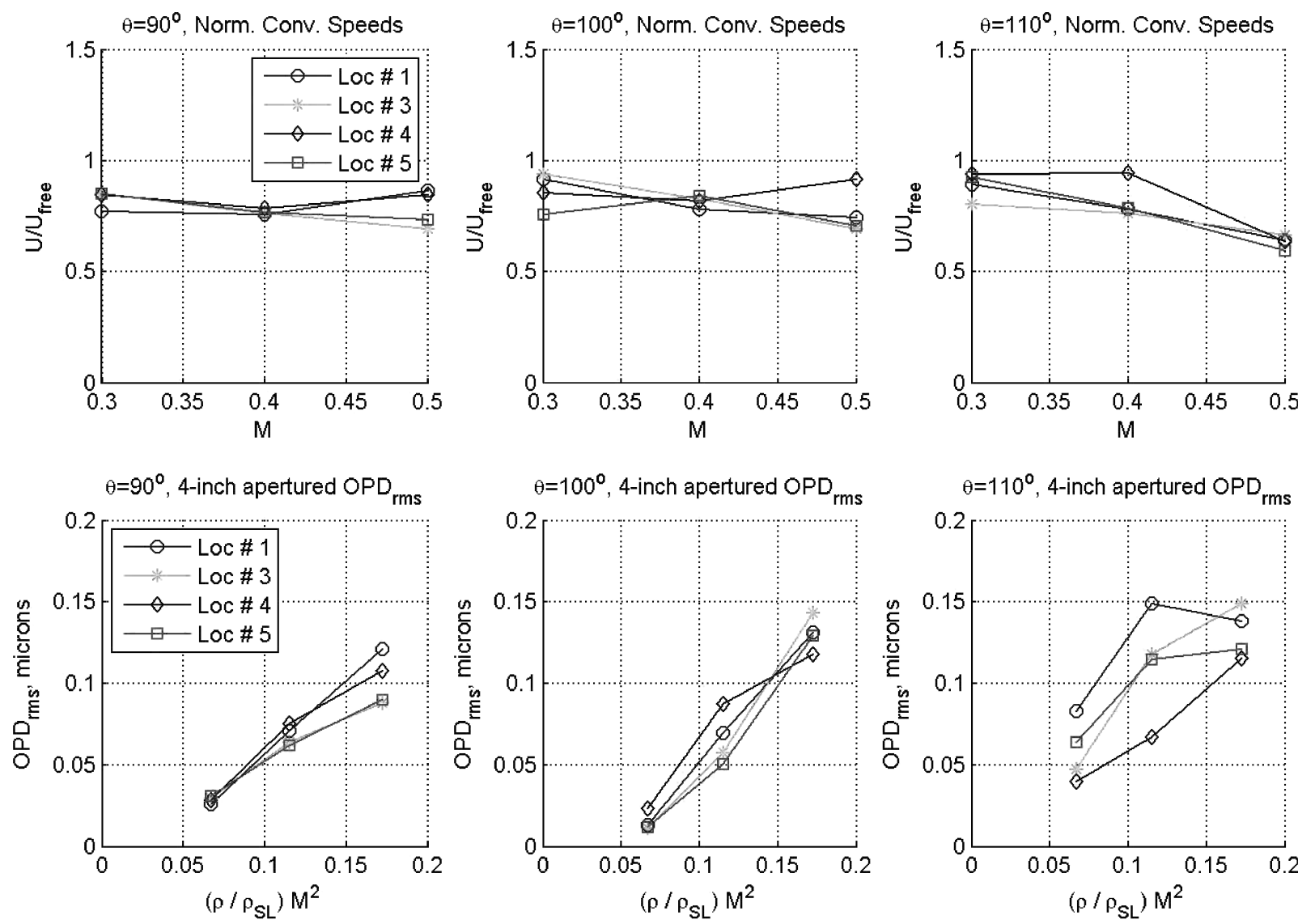

Fig. 10 Convective speeds (upper plots) and apertured $O P D_{\text {rms }}$ (lower plots) for different cases. 

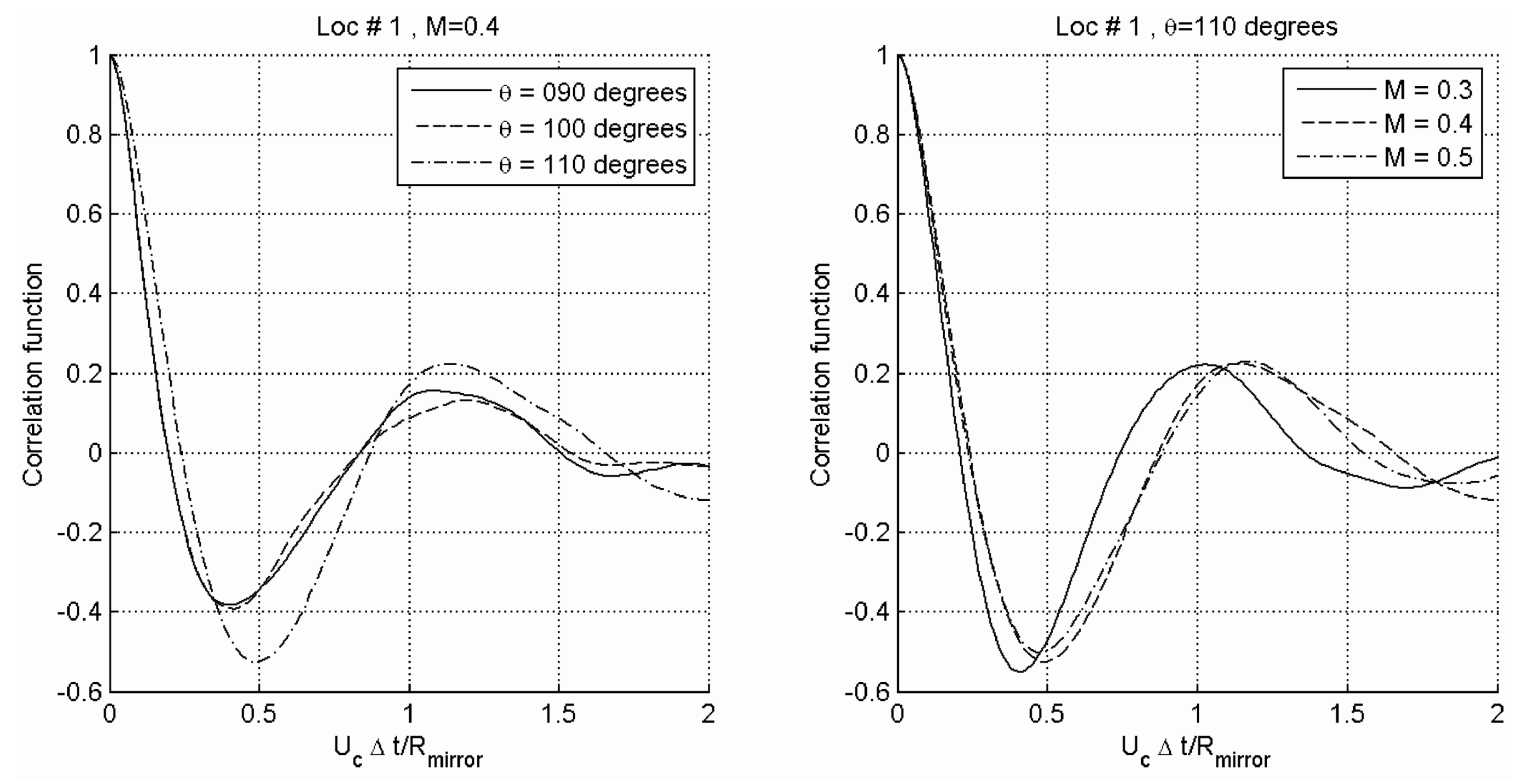

Fig. 11 OPD autocorrelation function for location 1.

given in Table 1 . OPD $_{\text {rms }}$ grows with the Mach number as $\mathrm{OPD}_{\mathrm{rms}} \sim$ $\rho / \rho_{\mathrm{SL}} M^{2}$ for most locations, except for the 100-deg case, for which the aberrations increase at a rate higher than $\sim \rho / \rho_{\mathrm{SL}} M^{2}$. One possible explanation is that the weak separation bubble over the window gets stronger with the Mach number, thus increasing its role in the optical aberrations. Although the overall aberration level increases with the Mach number, consistent with the findings of the 2-D wave fronts given in Table 1, specific locations show individual trends that are due to the complex interactions of the fluid mechanics over the window. This is particularly noticeable at location 1 for the $110-$ deg case, for which the $\mathrm{OPD}_{\mathrm{rms}}$ actually drops at $M=0.5$. The reason for this behavior is still not fully understood and may in part be due to the role of the breathing separation region, which may occur at less than $1 \mathrm{kHz}$ and, thus, is filtered out of the results. Overall, the $\mathrm{OPD}_{\mathrm{rms}} \sim \rho / \rho_{\mathrm{SL}} M^{2}$ trend is observed for most locations and can be used to scale the results for different Mach numbers and freestream densities.

Streamwise correlation lengths can be obtained from the Malley probe data by autocorrelating the OPD traces. Results for the middle location 1 at the window are presented in Fig. 11. In the left plot of Fig. 11, the Mach number was fixed at $M=0 . \overline{4}$ and the azimuthal angle was varied. If one uses the definition of the correlation length as the location of the first minima, then the correlation length at this location for $M=0.4$ increases from $0.4 R_{\text {mirror }}$ at $\theta=90$ and $100 \mathrm{deg}$ to $0.5 R_{\text {mirror }}$ at $\theta=110 \mathrm{deg}$. Similar trends were observed for all other locations. Although the weak growth in the structure is present, there is an uncanny similarity in not only the one structure but in the overall decay in correlation with subsequent structures. This is an important point; although we have noted that at 90 and $100 \mathrm{deg}$, the flow measurements indicate a boundary-layer-like flow, in fact, the role of the initial separation bubble must be significant. This bubble must be instrumental in forming cross-stream structures that subsequently convect with the turbulent reattached boundary layer. This "memory" of the separation region in the reattached boundary layer has been noticed in other flows [13].

In the right plot in Fig. 11, autocorrelation functions are plotted for different Mach numbers for the fixed azimuthal angle $\theta=110 \mathrm{deg}$. The correlation length is $0.4 R_{\text {mirror }}$ for $M=0.3$ and 0.4 , then slightly increases to $0.5 R_{\text {mirror }}$ for $M=0.5$. Inspection of the 2 -D wave fronts in Fig. 6 gives similar structure sizes in the streamwise direction.

The correlation length's independence with Mach numbers at 110 deg indicates that the flow is fully separated over the entire window; separated-shear-layer dynamics depend only on the adverse pressure gradient and are independent of the flow Reynolds number. But in the case of the weak separation and reattachment at 90 and $100 \mathrm{deg}$, the separation bubble is transient in nature and, therefore, is sensitive to the flow Reynolds number and the correlation length should vary with the Mach number. Indeed, this is the case, as can be seen in Fig. 12, in which the correlation length increases with the Mach number for both the 90 and 100-deg cases.

\section{Hot-Wire: Malley Probe Correlations}

Finally, the simultaneous measurements of the local velocity and the Malley probe were performed in an attempt to better understand the role that structures in the various regions of the flow have on the optical aberrations. These simultaneous Malley probe-velocity measurements were performed at locations 1 and 3 over the mirror, for the azimuthal angle of $110 \mathrm{deg}$ for one Mach number of $M=0.37$ (see Fig. 13). Note that the 110-deg case is fully separated flow over the window, as discussed in the previous section. The Malley probe beams were spaced horizontally, $5 \mathrm{~mm}$ apart, and the single hot wire was traversed in the normal direction, $3 \mathrm{~mm}$ downstream of the downstream beam. The sampling rate was $100 \mathrm{kHz}$, with a sampling time of $1 \mathrm{~s}$.

A zero-time-lag cross-correlation function between the velocity signal $u(z, t)$ and the downstream beam deflection angle $\delta_{2}(t)$ normalized by the maximum rms of the velocity, $u_{\mathrm{rms}}^{\mathrm{max}}$, and the rms of the deflection angle, $\delta_{2 \mathrm{rms}}$, was computed:

$$
\operatorname{Corr}(z)=\frac{\overline{u(z, t) \delta_{2}(t)}}{u_{\mathrm{rms}}^{\max } \delta_{2 \mathrm{rms}}}
$$

Results for both locations are shown in Fig. 14. At the mirror center location 1 , the correlation function exhibits relatively high positive values of $\sim 0.25$ on the low-speed side of the shear layer (i.e., closer to the mirror) for $z / R$ between 0.05 and 0.2 , with the peakcorrelation-function value of 0.3 at the maximum $u_{\text {rms }}$ location. Further away from the mirror, the correlation function quickly decays, but retains small negative values. These negative values are due to Biot-Savart-induced unsteady potential fluctuations in the freestream velocity field from the circulation density in the separated shear layer.

Further downstream, at location 3 , the correlation function has smaller values of $\sim 0.2$ and has a more localized peak centered at the middle of the shear layer $(z / R \sim 0.15)$. Lower correlation values may indicate a more turbulent or defused-vorticity flow with lesscrisp, optically aberrating structures.

To investigate the spectral range of significance, a Malley probevelocity correlation was computed at $z / r=0.13$, at which point the correlation function reaches its maximum; velocity and deflectionangle power spectra for locations 1 and 3 are presented in Fig. 15, in 

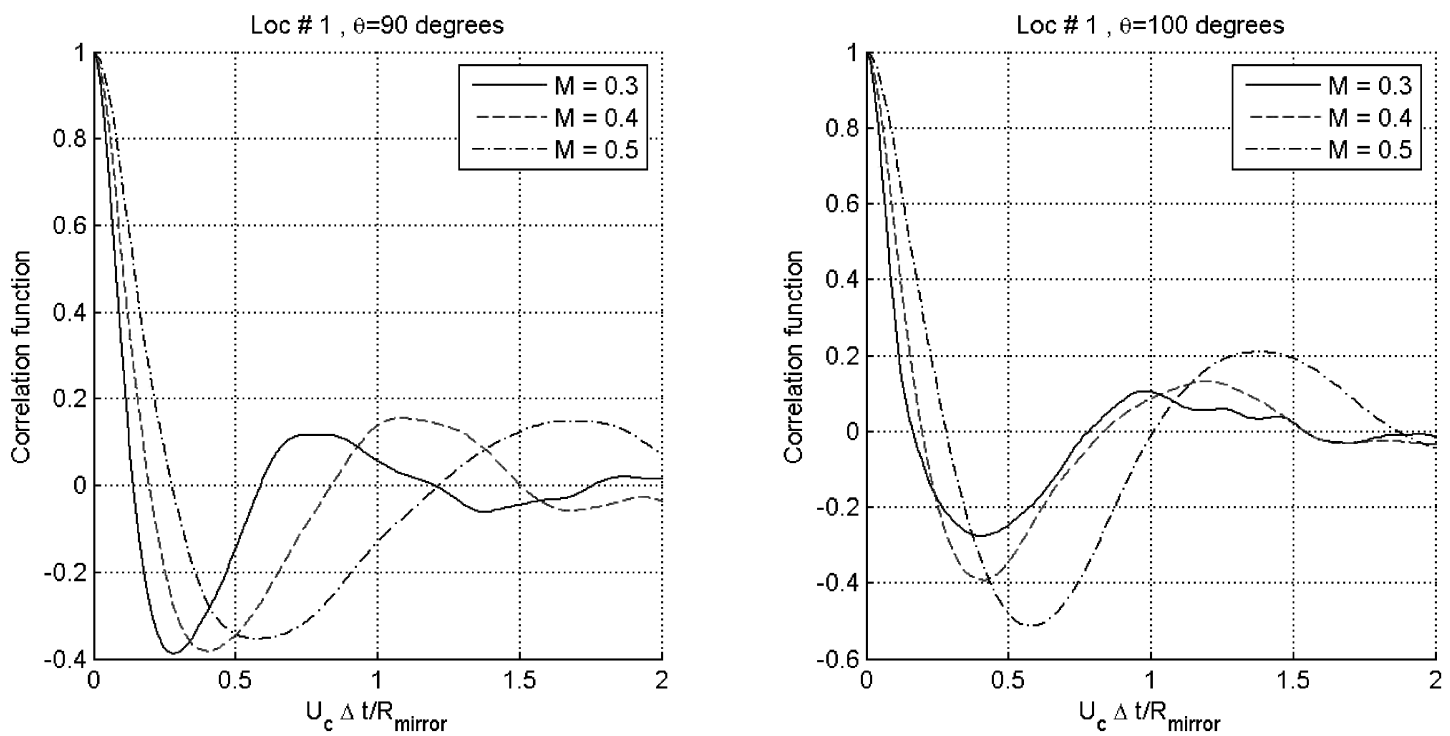

Fig. 12 OPD autocorrelation functions for location 1 for 90 and 100-deg cases.

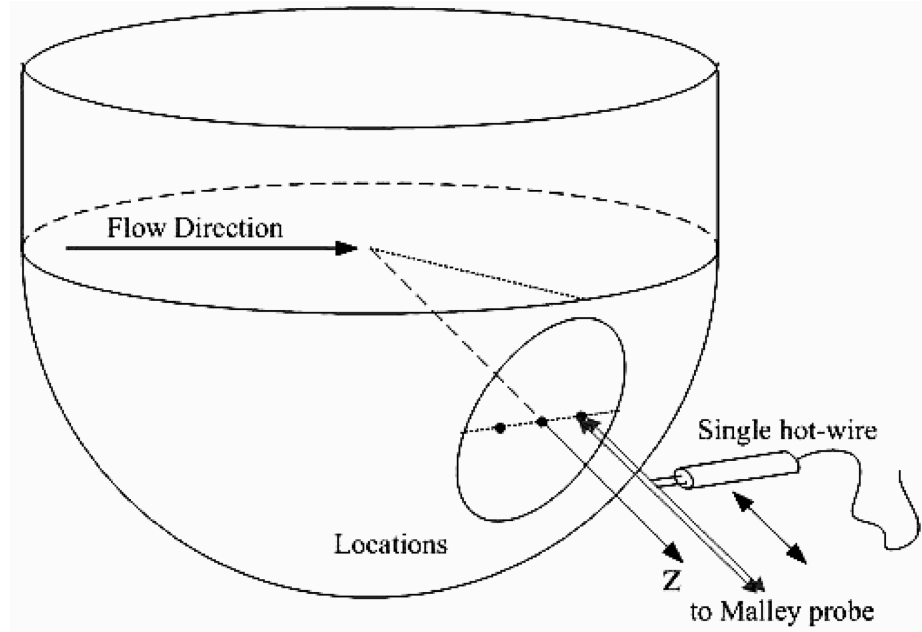

Fig. 13 Schematic of simultaneous Malley probe-velocity measurements.
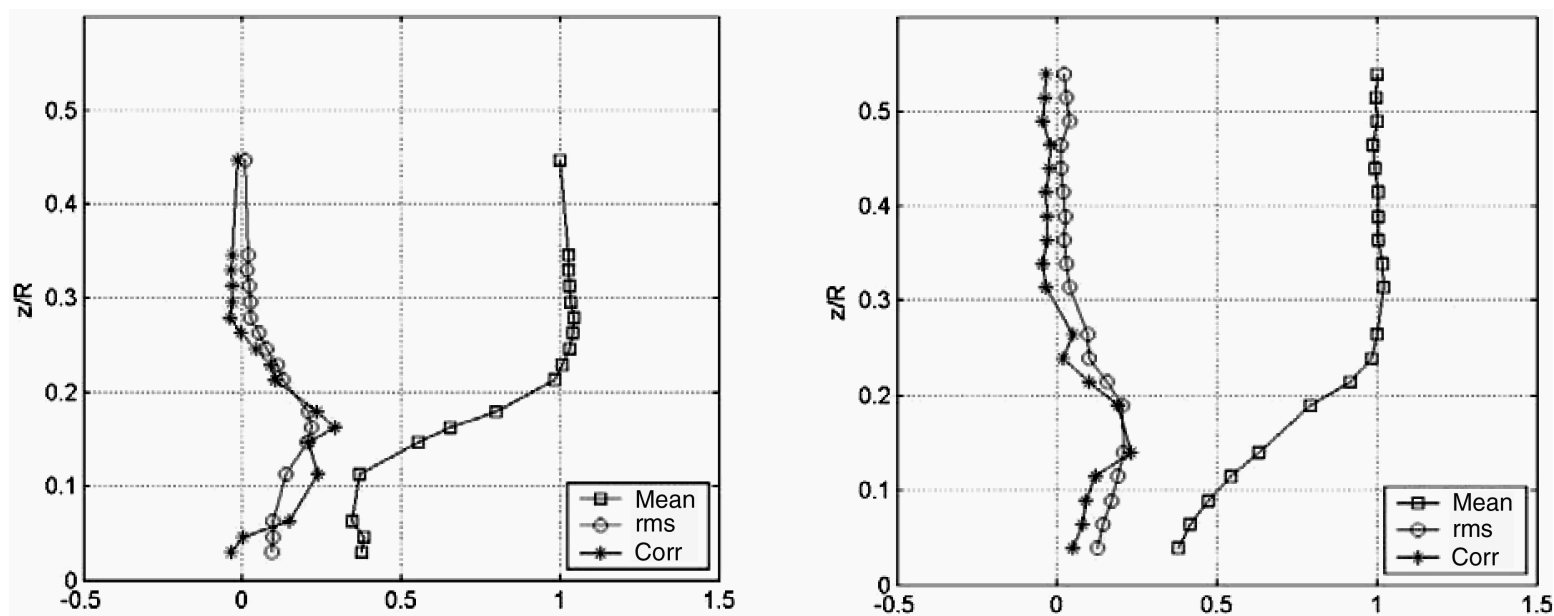

Fig. 14 Normalized velocity profiles and the correlation function for locations 1 (left) and 3 (right). Azimuthal angle is $110 \mathrm{deg}$ and freestream $M=0.37$.

the upper plots. The normalized spectral cross-correlation between the deflection angle and velocity for both locations is calculated and shown in Fig. 15, in the lower plots. Although there is no obvious peak in either the deflection-angle or velocity spectra, there is a strong peak in the normalized spectral cross-correlation at $1 \mathrm{kHz}$ for location 1 and at $1.3 \mathrm{kHz}$ at location 3 . Using these values of frequencies and taking convective speeds of $0.5 U_{\text {free }}$ for location 1 and $0.7 U_{\text {free }}$ for location 3 from Fig. 14, and assuming a convective 

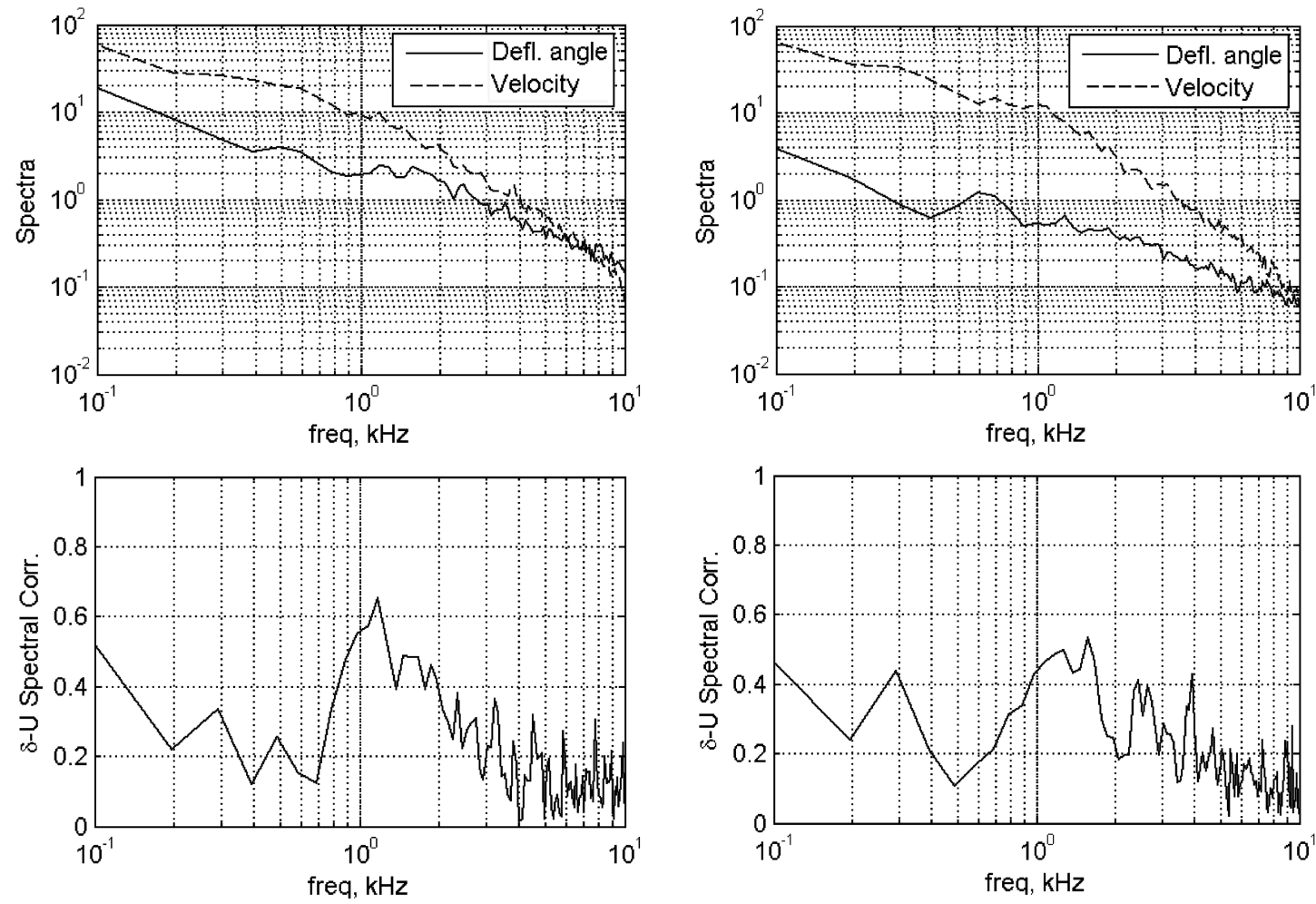

Fig. 15 Deflection angle and velocity spectra at $z / r=0.13$ and normalized spectral cross-correlation between deflection angle and velocity at $z / r=0.13$ for location 1 (left plots) and location 3 (right plots). Azimuthal angle is 110 deg and freestream is $M=0.37$.

nature of the structure, the structure correlation length was found to be approximately $0.07 \mathrm{~m}$, or $0.5 R$, which is consistent with the correlation length observed in Fig. 11, in the right plot.

The overall, nonzero correlation values indicate an important observation: the optically active region over the flat window is located on the low-speed side and the middle part of the shear layer. Additional studies should be conducted to see whether it is a general result for separated flows over the flat-windowed turrets or it is a function of view angle and other geometric parameters.

\section{Conclusions}

This paper presented an extensive initial effort to characterize levels of unsteady optical aberrations imposed on an outgoing, collimated laser beam from a hemisphere-on-cylinder turret arrangement with a flat window. Measurements at several azimuthal angles over a range of subsonic Mach numbers, at a fixed elevation angle, were performed. The optical measurements included both 2-D wave fronts and 1-D Malley probe data. Hot-wire measurements documented the velocity profiles in the normal direction from the flat window and have shown that the flow is separated over the flat window for the azimuthal angles bigger that $100 \mathrm{deg}$. Simultaneous velocity-Malley probe results reveal that the most optically active region in the separated flow over the flat window is in the shear-layer region, biased toward its low-speed side. This means that the convecting optical distortions over a flat window are governed mostly by the separated shear layer in an adverse-pressure-gradient environment, rather than the unsteady separation bubble formed behind the hemisphere, suggesting that the aberrating character of the flow should be receptive to flow-control devices placed upstream of the separation. Based on this observation, a variety of flow-control devices were successfully tested and shown to improve optical environment in the separated turbulent flow over the back-facing 2-D ramp [13] and in the flow over a simplified 2-D turret with a flat window [14].

Both $2-\mathrm{D}$ wave front measurements and properly apertured Malley probe results have revealed that the optical distortions get worse with increasing the azimuthal angle. Also, they grow with the incoming Mach numbers and the freestream density as $\rho / \rho_{\mathrm{SL}} M^{2}$. A similar trend was observed in other shear-layer-dominated flows $[11,14]$ and provides a very useful scaling law to compare data from different experiments or to extrapolate data to a different flow regime. To perform a correct comparison, a steady-lensing component and an instantaneous tip/tilt were removed in the postprocessing analysis from both the 2-D wave front data and the Malley probe results. Any stationary steady or unsteady lensing effects that were present were removed, although in different ways.

\section{Acknowledgments}

These efforts were sponsored by the U.S. Air Force Office of Scientific Research, Air Force Material Command, U.S. Air Force, under grant number F49620-03-1-0019. The U.S. government is authorized to reproduce and distribute reprints for governmental purposes, notwithstanding any copyright notation thereon.

\section{References}

[1] Gilbert, K. J., and Otten, L. J., Aero-Optical Phenomena, Vol. 80, Progress in Astronautics and Aeronautics, AIAA, New York, 1982.

[2] Jumper, E. J., and Fitzgerald, E. J., "Recent Advances in Aero-Optics," Progress in Aerospace Sciences, Vol. 37, No. 3, 2001, pp. 299-339.

[3] Hugo, R. J., Jumper, E. J., Havener, G., and Stepanek, C., "TimeResolved Wavefront Measurements Through a Compressible Free Shear Layer," AIAA Journal, Vol. 35, No. 4, 1997, pp. 671-677.

[4] Malley, M., Sutton, G. W., and Kincheloe, N., "Beam-Jitter Measurements for Turbulent Aero-Optical Path Differences," Applied Optics, Vol. 31, No. 22, Aug. 1992, pp. 4440-4443.

[5] Jumper, E. J., and Hugo, R. J., "Quantification of Aero-Optical Phase Distortion Using the Small-Aperture Beam Technique," AIAA Journal, Vol. 33, No. 11, 1995, pp. 2151-2157.

[6] Gordeyev, S., Jumper, E., Ng, T., and Cain, A., "Aero-Optical Characteristics of Compressible, Subsonic Turbulent Boundary Layers," AIAA Paper 2003-3606, 2003.

[7] Jumper, E. J., Gordeyev, S., Cavalieri, D., Ng, T., and Cain, A., "Methods and Systems for Measuring an Aberrated Wave Front," U.S. Patent Application No. 11/132,312, filed 19 May 2005.

[8] Duffin, D., Gordeyev, S., and Jumper, E., "Comparison of Wavefront Measurement Techniques on a Two-Dimensional Heated Jet," AIAA 
Paper 2004-2446, 2004.

[9] Craig, J. E., and Trolinger, J. D., "Propagation Diagnostic Technique for Turbulent Transonic Flow," AIAA Paper 84-0104, 1984.

[10] Siegenthaler, J., Gordeyev, S., and Jumper, E., "Mapping the Optically Aberrating Environment in a Partially Quieted Mach 0.6 Free Shear Layer," AIAA Paper 2003-3607, 2003.

[11] Gordeyev, S., Post, M., MacLaughlin, T., Ceniceros, J., and Jumper, E., "Survey of Optical Environment over Hemisphere-on-Cylinder Turret Using Suite of Wavefront Sensors," AIAA Paper 2006-3074, 2006.

[12] Siegenthaler, J., Gordeyev, S., and Jumper, E., "Shear Layers and
Aperture Effects for Aero-Optics," AIAA Paper 2005-4772, 2005.

[13] Gordeyev, S., Jumper, E., Ng, T., and Cain, A., "Optical Disturbances Caused by Transonic Separated Boundary Layer Behind a 20-Degree Ramp: Physics and Control," AIAA Paper 2004-0472, 2004.

[14] Gordeyev, S., Jumper, E., Ng, T., and Cain, A., "The Optical Environment of a Cylindrical Turret with a Flat Window and the Impact of Passive Control Devices,” AIAA Paper 2005-4657, 2005.

R. Lucht Associate Editor 